\title{
Analysis of Coupled Settlement by Using Three Asymptotic Expansion Parameters
}

\author{
John C.-C. Lü, ${ }^{1, *}$, Darwin Liao ${ }^{1}$ and Feng-Tsai Lin ${ }^{2}$ \\ ${ }^{1}$ Department of Civil Engineering, Chung Hua University, No. 707, Sec. 2, Wufu Rd., Hsinchu \\ 30012, Taiwan R.O.C. \\ ${ }^{2}$ Department of Naval Architecture and Ocean Engineering, National Kaohsiung University of \\ Science and Technology, No.142, Haijhuan Rd., Kaohsiung 81157, Taiwan R.O.C.
}

${ }^{*}$ Corresponding author

Keywords: Poroelasticity, Asymptotic expansion, Settlement.

\begin{abstract}
Based on Biot's coupled theory of poroelasticity, the uniform loading induced settlement is investigated by applying asymptotic expansion approach. Using three small asymptotic expansion parameters, the variables of settlement and excess pore fluid pressure are expressed in this study. Substituting these variables into the non-dimensional mathematical model, the coupled settlement model of the porous medium can be simplified into the uncoupled ones. The results are verified by the closed-form solutions to show the value of applying asymptotic expansion.
\end{abstract}

\section{Introduction}

Ground surface loading induced settlement is an important engineering point of issue. Many studies were focused on mechanical and hydraulic interactions due to coupled consolidation. Hydraulic seepage disturbance usually leads to a volumetric change of fluid and solid skeleton. The volumetric change of the porous stratum can increase excess pore fluid pressure and lead to a decrease in effective stress. The loss of shear resistance of solid skeleton may result in a hydraulic failure of the porous stratum. The simulation and its validation of this study is a major concern for the safety improvement of the engineering construction.

In the study of Yang and Tian [1], the hyperbolic functions for the asymptotic expansions of gamma function are given. Huang, Cao and Wang [2] aim to develop a simple and unified technique in finding asymptotic expansion of orthogonal polynomials from their difference equations. Barrera and Volkmer [3] give asymptotic expansion study of the strongly damped wave equation. A novel threescale asymptotic expansion used to evaluate viscoelastic analysis of composites with multiple configuration is systematically studied by Yang, Sun, Cui and Ge [4]. Kang [5] introduces and analyzes quadrature rules and asymptotic expansions of a few highly oscillatory infinite integrals. Hoang and Martinez [6] study the long-time behavior of spatially periodic solutions of the NavierStokes equations in the three-dimensional space. Yang [7] investigates asymptotic expansions for the Laplace transforms. An effective second-order reduced asymptotic expansion approach is proposed by Yang et al. [8] to analyze the thermo-mechanical coupling problems of nonlinear periodic heterogeneous materials.

The current study is concentrated on the analytical solutions of an isotropic pervious stratum subjected to uniform ground surface loading. The porous stratum is modelled as a linearly elastic medium with isotropic mechanical and hydraulic fluid flow properties. The analytical solutions of excess pore fluid pressure and displacements of the stratum due to uniform loading are obtained by using Laplace integral transforms. The results can provide better understanding of the uniform surface loading induced coupled responses of the pervious stratum. 


\section{Mathematical Model}

\section{Basic Equations}

Figure 1 illustrates a uniform surface loading on the pervious isotropic stratum. The stratum is modeled as a homogeneous elastic porous medium, and the constitutive law can thus be expressed by

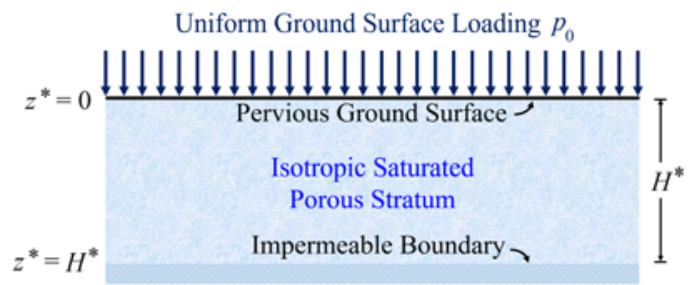

Figure 1. Consolidation due to uniform ground surface loading

$$
\begin{aligned}
& \tau_{i j}^{*}=2 G \varepsilon_{i j}^{*}+\frac{2 G v}{1-2 v} \varepsilon^{*} \delta_{i j}-\alpha p^{*} \delta_{i j}, i, j=1,2,3, \\
& \theta^{*}=\alpha \varepsilon^{*}+\frac{p^{*}}{Q},
\end{aligned}
$$

in which $\tau_{i j}^{*}, \varepsilon_{i j}^{*}, \theta^{*}, \varepsilon^{*}$ and $p^{*}$ are the total stress components, strain components, fluid content per unit volume of porous medium, volumetric strain and excess pore fluid pressure of the stratum, respectively. The symbols $G$ and $v$ are material constants of shear modulus and Poisson's ratio of the isotropic stratum. The coupled material constants $\alpha$ and $Q$ are defined by $\alpha=3\left(v_{u}-v\right) /\left[B(1-2 v)\left(1+v_{u}\right)\right]$ and $Q=2 G B^{2}\left(1+v_{u}\right)^{2} /\left[9\left(v_{u}-v\right)\left(1-2 v_{u}\right)\right]$, in which $B$ and $v_{u}$ are Skempton pore water pressure coefficient and undrained Poisson's ratio defined by Rice and Cleary [9]. The strain components $\varepsilon_{i j}^{*}$ are related to displacement components $u_{i}$ of the stratum as below:

$$
\varepsilon_{i j}^{*}=\frac{1}{2}\left(\frac{\partial u_{i}^{*}}{\partial x_{j}^{*}}+\frac{\partial u_{j}^{*}}{\partial x_{i}^{*}}\right), i, j=1,2,3
$$

In general, the total stresses $\tau_{i j}^{*}$ must satisfy the equilibrium equations of Newton's second law with the body forces $b_{i}^{*}$ :

$$
\tau_{i j, j}^{*}+b_{i}^{*}=0, i, j=1,2,3 .
$$

The equilibrium equations can be expressed in terms of displacements and excess pore fluid pressure as follows:

$$
G \nabla^{2} u_{i}^{*}+\frac{G}{1-2 v} \frac{\partial \varepsilon^{*}}{\partial x_{i}^{*}}-\alpha \frac{\partial p^{*}}{\partial x_{i}^{*}}=0, i=1,2,3
$$

in which $\nabla^{2}$ is the Laplacian operator. Let $v_{i}^{*}$ denotes the volumetric fluid flow through a unit area in a unit time. The hydraulic seepage flow should obey Darcy's law (6) and continuity equation (7) as shown below:

$$
\begin{aligned}
& v_{i}^{*}=-\frac{k}{\gamma_{w}} \frac{\partial p^{*}}{\partial x_{i}^{*}}, i=1,2,3, \\
& \frac{\partial \theta^{*}}{\partial t^{*}}=-\frac{\partial v_{1}^{*}}{\partial x_{1}^{*}}-\frac{\partial v_{2}^{*}}{\partial x_{2}^{*}}-\frac{\partial v_{3}^{*}}{\partial x_{3}^{*}},
\end{aligned}
$$


in which the symbols $k$ and $\gamma_{w}$ are permeability and unit weight of pore water of the porous medium. The governing equation for fluid flow can be derived by using Darcy's law (6) and continuity equation (7) as below:

$$
\frac{k}{\gamma_{w}} \nabla^{2} p^{*}=\alpha \frac{\partial \varepsilon^{*}}{\partial t^{*}}+\frac{1}{Q} \frac{\partial p^{*}}{\partial t^{*}} .
$$

The governing equations (5) and (8) can be simplified as following if one-dimensional consolidation is considered:

$$
\begin{aligned}
& \frac{1}{a} \frac{\partial^{2} u_{3}^{*}}{\partial x_{3}^{* 2}}-\alpha \frac{\partial p^{*}}{\partial x_{3}^{*}}=0, \\
& \frac{k}{\gamma_{w}} \frac{\partial^{2} p^{*}}{\partial x_{3}^{* 2}}=\alpha \frac{\partial^{2} u_{3}^{*}}{\partial x_{3}^{*} \partial t^{*}}+\frac{1}{Q} \frac{\partial p^{*}}{\partial t^{*}},
\end{aligned}
$$

in which the final compressibility $a$ is defined as $a=(1-2 v) /[2 G(1-v)]$.

\section{Boundary Conditions and Initial Conditions}

As shown in Figure 1, the study can be treated as one-dimensional consolidation model for the uniform ground surface loading. The soil layer of ground surface boundary $x_{3}^{*}=0$ is treated as pervious for all times, while the lower boundary at $x_{3}^{*}=H^{*}$ is assumed as impervious with a rigid rock shown below, i.e.:

$$
\begin{aligned}
& p^{*}\left(0, t^{*}\right)=0, \frac{\partial u_{3}^{*}\left(0, t^{*}\right)}{\partial x_{3}^{*}}=-a p_{0}, \\
& \frac{\partial p^{*}\left(H^{*}, t^{*}\right)}{\partial x_{3}^{*}}=0, u_{3}^{*}\left(H^{*}, t^{*}\right)=0 .
\end{aligned}
$$

The initial conditions can be treated as [10]:

$$
\begin{aligned}
& p^{*}\left(x_{3}^{*}, 0^{+}\right)=\frac{a-a_{i}}{\alpha a} p_{0}, \\
& \frac{\partial u_{3}^{*}\left(x_{3}^{*}, 0^{+}\right)}{\partial x_{3}^{*}}=-a_{i} p_{0},
\end{aligned}
$$

where $a_{i}$ is the instantaneous compressibility, and $a_{i}=a /\left(1+\alpha^{2} a Q\right)$. If the compressibility of solid skeleton and pore water can be neglected, then $\alpha=1, Q \rightarrow \infty$ and $a_{i}=0$ [10].

\section{Asymptotic Expansion}

Using three small asymptotic expansion parameters ( $\varepsilon=\sigma_{0} / G, \varepsilon=\sigma_{0} / Q$ and $\varepsilon=\alpha \gamma_{w} H^{*} / G$ ), the space variable $x_{3}^{*}$, time variable $t^{*}$, vertical displacement $u_{3}^{*}$ and excess pore fluid pressure $p^{*}$ can be expressed as

$$
z=\frac{x_{3}^{*}}{H^{*}}, t=\frac{t^{*}}{t_{0}}, w=\frac{u_{3}^{*}}{u_{0}}, p=\frac{p^{*}}{\sigma_{0}} .
$$

The parameters $H^{*}, \sigma_{0}, u_{0}$ and $t_{0}$ are layered stratum thickness, critical excess pore fluid pressure, characteristic displacement and characteristic time as the non-dimensional parameters, respectively. 
The one-dimensional consolidation settlement and excess pore fluid pressure can be expressed as below based on the asymptotic expansion theory:

$$
\begin{aligned}
& w=\varepsilon^{0} w^{(0)}+\varepsilon^{1} w^{(1)}+\varepsilon^{2} w^{(2)}+\varepsilon^{3} w^{(3)}+\cdots, \\
& p=\varepsilon^{0} p^{(0)}+\varepsilon^{1} p^{(1)}+\varepsilon^{2} p^{(2)}+\varepsilon^{3} p^{(3)}+\cdots,
\end{aligned}
$$

where $w$ and $p$ are the dimensionless vertical displacement and excess pore fluid pressure, respectively. The symbols $w^{(i)}$ and $p^{(i)}$ are the $n$ th-order dimensionless vertical displacement and excess pore fluid pressure with respect to the asymptotic expansion parameter $\varepsilon^{i}$.

\section{Using Asymptotic Expansion Parameter $\varepsilon=\sigma_{0} / G$}

Therefore, the characteristic parameters should be defined as $u_{0}=a \sigma_{0} H^{*}, t_{0}=\gamma_{w} H^{* 2} / k \sigma_{0}$ and $\sigma_{0}=10^{-3} G$ [11]. Then the governing equations of (9) and (10) can be transformed to:

$$
\begin{aligned}
& \frac{\partial^{2} w}{\partial z^{2}}-\alpha \frac{\partial p}{\partial z}=0, \\
& \frac{\partial^{2} p}{\partial z^{2}}-\alpha a \sigma_{0} \frac{\partial^{2} w}{\partial z \partial t}-\frac{\sigma_{0}}{Q} \frac{\partial p}{\partial t}=0 .
\end{aligned}
$$

Besides, the transformed boundary conditions and initial conditions can be expressed as:

$$
\begin{aligned}
& p(0, t)=0, \frac{\partial w(0, t)}{\partial z}=-\frac{p_{0}}{\sigma_{0}}, \frac{\partial p(1, t)}{\partial z}=0, w(1, t)=0, \\
& p\left(z, 0^{+}\right)=\frac{a-a_{i}}{\alpha a} \frac{p_{0}}{\sigma_{0}}, \frac{\partial w\left(z, 0^{+}\right)}{\partial z}=-\frac{a_{i} p_{0}}{a \sigma_{0}} .
\end{aligned}
$$

Based on equations (16) and (17), the transformed governing equations, boundary conditions and initial conditions are derived as following.

- Transformed governing equations of order $\varepsilon^{0}$

$$
\begin{aligned}
& \frac{\partial^{2} w^{(0)}}{\partial z^{2}}-\alpha \frac{\partial p^{(0)}}{\partial z}=0, \\
& \frac{\partial^{2} p^{(0)}}{\partial z^{2}}-\frac{\sigma_{0}}{Q} \frac{\partial p^{(0)}}{\partial t}=0 .
\end{aligned}
$$

- Transformed boundary conditions and initial conditions of order $\varepsilon^{0}$

$$
\begin{aligned}
& p^{(0)}(0, t)=0, \frac{\partial w^{(0)}(0, t)}{\partial z}=-\frac{p_{0}}{\sigma_{0}}, \frac{\partial p^{(0)}(1, t)}{\partial z}=0, w^{(0)}(1, t)=0, \\
& p^{(0)}\left(z, 0^{+}\right)=\frac{a-a_{i}}{\alpha a} \frac{p_{0}}{\sigma_{0}}, \frac{\partial w^{(0)}\left(z, 0^{+}\right)}{\partial z}=-\frac{a_{i} p_{0}}{a \sigma_{0}} .
\end{aligned}
$$

- $\quad$ Transformed governing equations of order $\varepsilon^{1}$

$$
\frac{\partial^{2} w^{(1)}}{\partial z^{2}}-\alpha \frac{\partial p^{(1)}}{\partial z}=0
$$




$$
\frac{\partial^{2} p^{(1)}}{\partial z^{2}}-\frac{\sigma_{0}}{Q} \frac{\partial p^{(1)}}{\partial t}=\frac{\alpha}{2 \eta} \frac{\partial^{2} w^{(0)}}{\partial z \partial t}
$$

- Transformed boundary conditions and initial conditions of order $\varepsilon^{1}$

$$
\begin{aligned}
& p^{(1)}(0, t)=0, \frac{\partial w^{(1)}(0, t)}{\partial z}=0, \frac{\partial p^{(1)}(1, t)}{\partial z}=0, w^{(1)}(1, t)=0 . \\
& p^{(1)}\left(z, 0^{+}\right)=0, \frac{\partial w^{(1)}\left(z, 0^{+}\right)}{\partial z}=0 .
\end{aligned}
$$

The transformed mathematical model is uncoupled as shown in the governing equations (22), (23), (26) and (27).

\section{Using Asymptotic Expansion Parameter $\varepsilon=\sigma_{0} / Q$}

The transformed mathematical model can be expressed in the same form with chosen asymptotic parameter $\varepsilon=\sigma_{0} / G$. Besides, the characteristic parameters can also be defined as $u_{0}=a \sigma_{0} H^{*}$, $t_{0}=\gamma_{w} H^{* 2} / k \sigma_{0}$ and $\sigma_{0}=10^{-3} G$ [11]. Then the transformed governing equations are derived in (30)-(33) while the transformed boundary conditions and initial conditions are found in the same form as (24)(25) and (28)-(29).

- Transformed governing equations of order $\varepsilon^{0}$

$$
\begin{aligned}
& \frac{\partial^{2} w^{(0)}}{\partial z^{2}}-\alpha \frac{\partial p^{(0)}}{\partial z}=0, \\
& \frac{\partial^{3} w^{(0)}}{\partial z^{3}}-\alpha^{2} a \sigma_{0} \frac{\partial^{2} w^{(0)}}{\partial z \partial t}=0 .
\end{aligned}
$$

- $\quad$ Transformed governing equations of order $\varepsilon^{1}$

$$
\begin{aligned}
& \frac{\partial^{2} w^{(1)}}{\partial z^{2}}-\alpha \frac{\partial p^{(1)}}{\partial z}=0, \\
& \frac{\partial^{3} w^{(1)}}{\partial z^{3}}-\alpha^{2} a \sigma_{0} \frac{\partial^{2} w^{(1)}}{\partial z \partial t}=\alpha \frac{\partial p^{(0)}}{\partial t} .
\end{aligned}
$$

Again, the transformed mathematical model is uncoupled as expressed in the transformed governing equations (30)-(33).

\section{Using Asymptotic Expansion Parameter $\varepsilon=\alpha \gamma_{w} H^{*} / G$}

Based on the asymptotic expansion parameter $\varepsilon=\alpha \gamma_{w} H^{*} / G$, and the defined characteristic parameters $u_{0}=H^{*}, t_{0}=H^{*} / k$, the governing equations (9)-(10) can be transformed to:

$$
\begin{aligned}
& \frac{\partial^{2} w}{\partial z^{2}}-\alpha a G \frac{\partial p}{\partial z}=0, \\
& \frac{\partial^{2} p}{\partial z^{2}}-\frac{\alpha \gamma_{w} H^{*}}{G} \frac{\partial^{2} w}{\partial z \partial t}-\frac{\gamma_{w} H^{*}}{Q} \frac{\partial p}{\partial t}=0 .
\end{aligned}
$$

The corresponding boundary conditions and initial conditions can be transformed as below:

$$
p(0, t)=0, \frac{\partial w(0, t)}{\partial z}=-a p_{0}, \frac{\partial p(1, t)}{\partial z}=0, w(1, t)=0,
$$




$$
p\left(z, 0^{+}\right)=\frac{a-a_{i}}{\alpha a} \frac{p_{0}}{G}, \frac{\partial w\left(z, 0^{+}\right)}{\partial z}=-a_{i} p_{0} .
$$

The corresponding transformed governing equations, boundary conditions and initial conditions are derived as below.

- Transformed governing equations of order $\varepsilon^{0}$

$$
\begin{gathered}
\frac{\partial^{2} w^{(0)}}{\partial z^{2}}-a \alpha G \frac{\partial p^{(0)}}{\partial z}=0, \\
\frac{\partial^{2} p^{(0)}}{\partial z^{2}}-\frac{\gamma_{w} H^{*}}{Q} \frac{\partial p^{(0)}}{\partial t}=0 .
\end{gathered}
$$

- Transformed boundary conditions and initial conditions of order $\varepsilon^{0}$

$$
\begin{aligned}
& p^{(0)}(0, t)=0, \frac{\partial w^{(0)}(0, t)}{\partial z}=-a p_{0}, \frac{\partial p^{(0)}(1, t)}{\partial z}=0, w^{(0)}(1, t)=0, \\
& p^{(0)}\left(z, 0^{+}\right)=\frac{a-a_{i}}{\alpha a} \frac{p_{0}}{G}, \frac{\partial w^{(0)}\left(z, 0^{+}\right)}{\partial z}=-a_{i} p_{0} .
\end{aligned}
$$

- Transformed governing equations of order $\varepsilon^{1}$

$$
\begin{aligned}
& \frac{\partial^{2} w^{(1)}}{\partial z^{2}}-a \alpha G \frac{\partial p^{(1)}}{\partial z}=0, \\
& \frac{\partial^{2} p^{(1)}}{\partial z^{2}}-\frac{\gamma_{w} H^{*}}{Q} \frac{\partial p^{(1)}}{\partial t}=\frac{\alpha \gamma_{w} H^{*}}{G} \frac{\partial^{2} w^{(0)}}{\partial z \partial t} .
\end{aligned}
$$

- Transformed boundary conditions and initial conditions of order $\varepsilon^{1}$

$$
\begin{aligned}
& p^{(1)}(0, t)=0, \frac{\partial w^{(1)}(0, t)}{\partial z}=0, \frac{\partial p^{(1)}(1, t)}{\partial z}=0, w^{(1)}(1, t)=0, \\
& p^{(1)}\left(z, 0^{+}\right)=0, \frac{\partial w^{(1)}\left(z, 0^{+}\right)}{\partial z}=0 .
\end{aligned}
$$

The mathematical model is decoupled as shown in the transformed governing equations (38), (39), (42) and (43).

\section{Analytical Solutions}

The closed-form solutions of the uniform loading induced consolidation settlement was derived by Biot [10] and can be expressed in the non-dimensional form:

$$
\begin{aligned}
& w=\frac{8\left(a-a_{i}\right) p_{0}}{a \pi^{2} \sigma_{0}} \times \sum_{n=1}^{\infty} \frac{1}{(2 n-1)^{2}}\left\{1-\exp \left[-\frac{(2 n-1)^{2} \pi^{2}}{4\left(1+\alpha^{2} a Q\right)}\left(\frac{Q}{\sigma_{0}}\right)^{2} t\right]\right\}, \\
& p=\frac{4\left(a-a_{i}\right) p_{0}}{\alpha a \pi \sigma_{0}} \times \sum_{n=1}^{\infty} \frac{1}{2 n-1} \exp \left[-\frac{(2 n-1)^{2} \pi^{2}}{4\left(1+\alpha^{2} a Q\right)}\left(\frac{Q}{\sigma_{0}}\right)^{2} t\right] \sin \frac{(2 n-1) \pi z}{2} .
\end{aligned}
$$


The analytical solutions of transformed mathematical model of order $\varepsilon^{i}$ can be derived as $w \cong w^{(0)}+\varepsilon w^{(1)}$ and $p \cong p^{(0)}+\varepsilon p^{(1)}$ by using Laplace transform technique. After a proper manipulation, the analytical solutions are derived as equations in (48)-(53).

Using Asymptotic Expansion Parameter $\varepsilon=\sigma_{0} / G$

$$
\begin{aligned}
& w \cong \frac{p_{0}}{\sigma_{0}}\left\{1-z-\sum_{n=1}^{\infty} \frac{a-a_{i}}{\pi^{2} a}\left[\frac{8}{(2 n-1)^{2}}+\varepsilon \frac{\pi^{2} \alpha^{2} Q}{\eta \sigma_{0}} c_{1} t\right] \times \exp \left[-\frac{(2 n-1)^{2} \pi^{2}}{4} c_{1} t\right] \cos \frac{(2 n-1) \pi z}{2}\right\}, \\
& p \cong \frac{a-a_{i}}{\pi a} \frac{p_{0}}{\sigma_{0}} \sum_{n=1}^{\infty}\left[\frac{4}{\alpha(2 n-1)}+\varepsilon \frac{(2 n-1) \pi^{2} \alpha Q}{2 \eta \sigma_{0}} c_{1} t\right] \times \exp \left[-\frac{(2 n-1)^{2} \pi^{2}}{4} c_{1} t\right] \sin \frac{(2 n-1) \pi z}{2},
\end{aligned}
$$

where $c_{1}=\frac{Q}{\sigma_{0}}=\frac{2 B^{2}(1-2 v)\left(1+v_{u}^{2}\right)^{2}}{9\left(v_{u}-v\right)\left(1-2 v_{u}\right)} \frac{G}{\sigma_{0}}$ and $\eta=\frac{1-v}{1-2 v}$.

\section{Using Asymptotic Expansion Parameter $\varepsilon=\sigma_{0} / Q$}

$$
\begin{aligned}
& w \cong \frac{p_{0}}{\sigma_{0}}\left\{1-z+\sum_{n=1}^{\infty} \frac{a-a_{i}}{\pi^{2} a} \frac{8(-1)^{n}}{(2 n-1)^{2}} \times \exp \left[-\frac{(2 n-1)^{2} \pi^{2}}{4} c_{2} t\right] \sin \frac{(2 n-1) \pi(1-z)}{2}\right. \\
& +\varepsilon\left\langle\frac { \pi ( a - a _ { i } ) } { 2 a } c _ { 2 } ^ { 2 } \int _ { 0 } ^ { t } \left[ 1+\sum_{n=1}^{\infty} \frac{4}{\pi} \frac{(-1)^{n}}{2 n-1} \times \exp \left(-\frac{(2 n-1)^{2} \pi^{2}}{4} c_{2} u\right) \cos \frac{(2 n-1) \pi z}{2}\right.\right. \\
& \times \sum_{n=1}^{\infty}(-1)^{n-1}(2 n-1) \exp \left(-\frac{(2 n-1)^{2} \pi^{2}}{4} c_{2}(t-u)\right] d u-c_{2}\left[1-z-\frac{8}{\pi^{2}} \sum_{n=1}^{\infty} \frac{(-1)^{n}}{(2 n-1)^{3}} \exp \left(-\frac{(2 n-1)^{2} \pi^{2}}{4} c_{2} t\right)\right. \\
& \left.\left.\left.\times \sin \frac{(2 n-1) \pi(z-1)}{2}\right]-c_{2} z\left[1+\frac{4}{\pi} \sum_{n=1}^{\infty} \frac{(-1)^{n}}{2 n-1} \times \exp \left(-\frac{(2 n-1)^{2} \pi^{2}}{2} c_{2} t\right) \cos \frac{(2 n-1) \pi(1-z)}{2}\right]\right)\right\}, \\
& p \cong \frac{p_{0}}{\sigma_{0}}\left\{-\frac{4\left(a-a_{i}\right)}{\pi a \alpha} \sum_{n=1}^{\infty} \frac{(-1)^{n}}{2 n-1} \times \exp \left(-\frac{(2 n-1)^{2} \pi^{2}}{4} c_{2} t\right) \cos \frac{(2 n-1) \pi(1-z)}{2}\right. \\
& +\varepsilon\left\langle\frac { a - a _ { i } } { 2 a } \left[ 2 \pi c_{2}^{2} \exp \left(-\frac{(2 n-1)^{2} \pi^{2}}{4} c_{2} t\right) \times \int_{0}^{t}(-1)^{n-1} \exp \left(-\frac{(2 n-1)^{2} \pi^{2}}{4} c_{2} u\right) \sin \frac{(2 n-1) \pi z}{2}\right.\right. \\
& \times \sum_{n=1}^{\infty}(-1)^{n-1}(2 n-1) \exp \left(-\frac{(2 n-1)^{2} \pi^{2}}{4} c_{2} u\right) d u-2 c_{2} z \sum_{n=1}^{\infty}(-1)^{n-1} \exp \left(-\frac{(2 n-1)^{2} \pi^{2}}{4} c_{2} t\right) \\
& \left.\left.\times \sin \frac{(2 n-1) \pi(z-1)}{2}\right\rangle\right\},
\end{aligned}
$$

where the symbol $c_{2}=1 / \alpha^{2} a \sigma_{0}$.

Using Asymptotic Expansion Parameter $\varepsilon=\alpha \gamma_{w} H^{*} / G$

$$
w \cong a p_{0}\left\{1-z-2\left(a-a_{i}\right) \sum_{n=1}^{\infty}\left[\frac{4}{\pi^{2} a(2 n-1)^{2}}+\varepsilon \alpha^{2} Q c_{3} t\right] \times \exp \left(-\frac{(2 n-1)^{2} \pi^{2}}{4} c_{3} t\right) \cos \frac{(2 n-1) \pi z}{2}\right\},
$$




$$
\left.p \cong \frac{\left(a-a_{i}\right) p_{0}}{G} \sum_{n=1}^{\infty}\left[\frac{4}{\pi a \alpha(2 n-1)}+\varepsilon(2 n-1) \pi \alpha Q c_{3} t\right] \times \exp \left(-\frac{(2 n-1)^{2} \pi^{2}}{4} c_{3} t\right) \sin \frac{(2 n-1) \pi z}{2}\right\},
$$

in which $c_{3}=Q / \gamma_{w} H^{*}$.

\section{Numerical Results}

The model input parameters (Charcoal granite, Tennessee marble and Ruhr sandstone) are given in Table I, following Detournay and Cheng [12]. The normalized settlement $w^{(0)}$ and normalized excess pore fluid pressure $p^{(0)}$ are defined as the zeroth order solutions, respectively. The approximate solution of normalized settlement is written as $w \cong w^{(0)}+\varepsilon w^{(1)}$, while the approximate solution of normalized excess pore fluid pressure is determined by $p \cong p^{(0)}+\varepsilon p^{(1)}$. The theoretical solutions of the uniform loading induced normalized consolidation settlement $w$ and normalized excess pore fluid pressure $p$ were derived by Biot [10] as expressed in (46) and (47), respectively. The zeroth order solutions, approximate solutions, theoretical solutions of normalized settlement and excess pore pressure dissipation are employed to numerically display in Figures II to VII.

Table 1. Model parameters [12]

\begin{tabular}{|c|c|c|c|}
\hline \multirow{2}{*}{ Category } & \multicolumn{3}{|c|}{ Parameters } \\
\cline { 2 - 4 } & $\begin{array}{c}\text { Poisson's Ratio } \\
v\end{array}$ & $\begin{array}{c}\text { Undrained } \\
\text { Poisson's Ratio } \\
v_{u}\end{array}$ & $\begin{array}{c}\text { Skempton } \\
\text { Pore Fluid } \\
\text { Pressure } \\
\text { Coefficient } \\
B\end{array}$ \\
\hline Case 1: Charcoal Granite & 0.27 & 0.30 & 0.55 \\
\hline Case 2: Tennessee Marble & 0.25 & 0.27 & 0.51 \\
\hline Case 3: Ruhr Sandstone & 0.12 & 0.31 & 0.88 \\
\hline
\end{tabular}

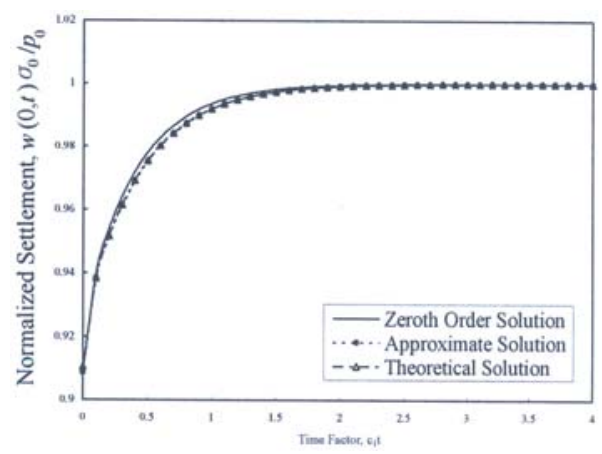

Figure 2(a). Comparison of normalized settlement of case 1: $v=0.27, v_{u}=0.30, B=0.55 ; \varepsilon=\sigma_{0} / G, c_{1}=Q / \sigma_{0}$.

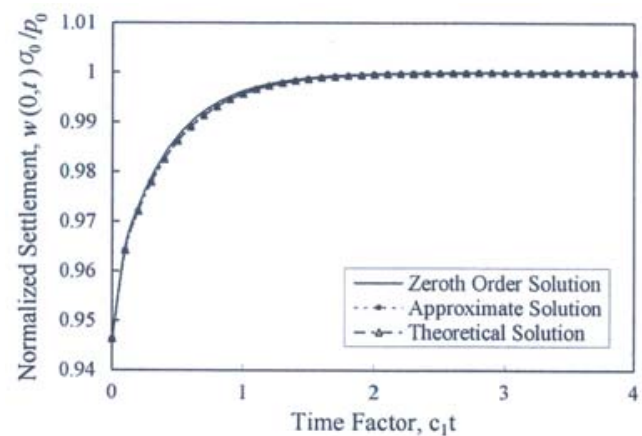

Figure 2(b). Comparison of normalized settlement of case 2: $v=0.25, v_{u}=0.27, B=0.51 ; \varepsilon=\sigma_{0} / G, c_{1}=Q / \sigma_{0}$. 


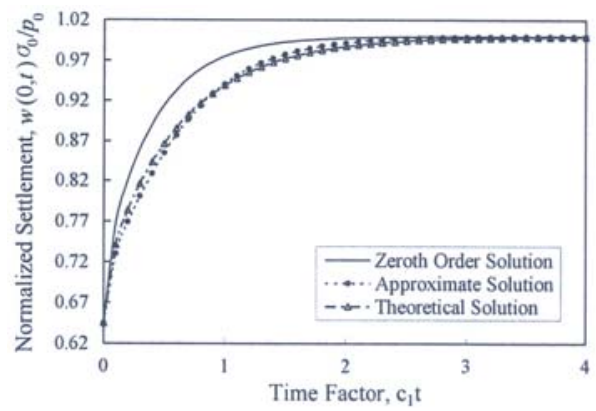

Figure 2(c). Comparison of normalized settlement of case 3: $v=0.12, v_{u}=0.31, B=0.88 ; \varepsilon=\sigma_{0} / G, c_{1}=Q / \sigma_{0}$.

In this paper, three cases of Charcoal granite, Tennessee marble and Ruhr sandstone are simulated to examine the effects of three asymptotic expansion solutions. Based on asymptotic expansion parameter $\varepsilon=\sigma_{0} / G$, the three cases of approximate solutions of increasing normalized settlements $w \cong w^{(0)}+\varepsilon w^{(1)}$ and excess pore pressure dissipation $p \cong p^{(0)}+\varepsilon p^{(1)}$ are closed to theoretical solutions as shown in Figures II and III. However, the simulated results of Charcoal granite and Tennessee marble are better than the simulation effects of Ruhr sandstone.

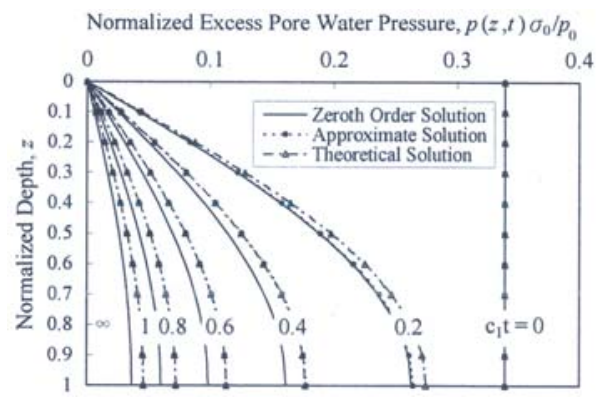

Figure 3(a). Comparison of normalized excess pore fluid pressure of case 1: $v=0.27, v_{u}=0.30, B=0.55 ; \varepsilon=\sigma_{0} / G$, $c_{1}=Q / \sigma_{0}$

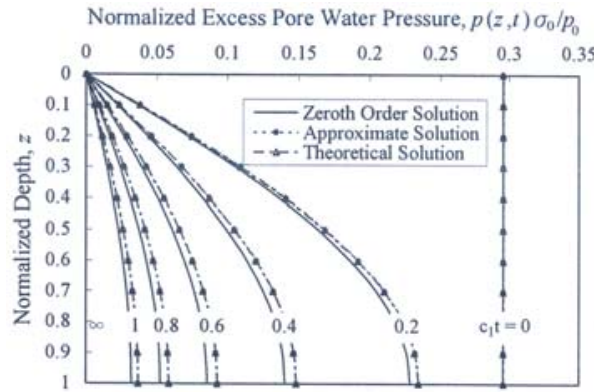

Figure 3(b). Comparison of normalized excess pore fluid pressure of case 2: $v=0.25, v_{u}=0.27, B=0.51 ; \varepsilon=\sigma_{0} / G$, $c_{1}=Q / \sigma_{0}$.

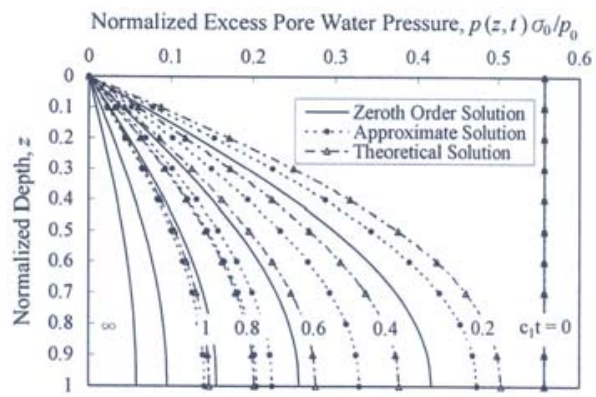

Figure 3(c). Comparison of normalized excess pore fluid pressure of case 3: $v=0.12, v_{u}=0.31, B=0.88 ; \varepsilon=\sigma_{0} / G$, $c_{1}=Q / \sigma_{0}$ 
Figures IV and V show the normalized settlements and excess pore pressure dissipation on the basis of asymptotic expansion parameter $\varepsilon=\sigma_{0} / Q$. As observed in Figure IV, the zeroth order solutions and approximate solutions of normalized settlements are very close to the theoretical solutions. It should be underlined that the excess pore pressure simulations of Charcoal granite stratum are good enough as shown in Figure V(a). Usually, the long-term simulation of excess pore pressure is better than shortterm simulation as illustrated in Figures V(b) and V(c).

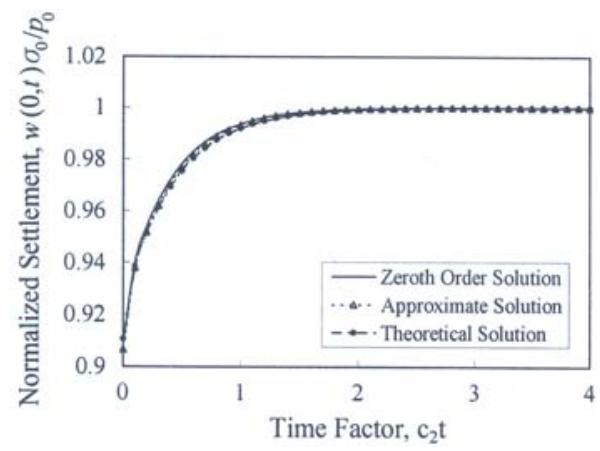

Figure 4(a). Comparison of normalized settlement of case 1: $v=0.27, v_{u}=0.30, B=0.55 ; \varepsilon=\sigma_{0} / Q, c_{2}=1 / \alpha^{2} a \sigma_{0}$.

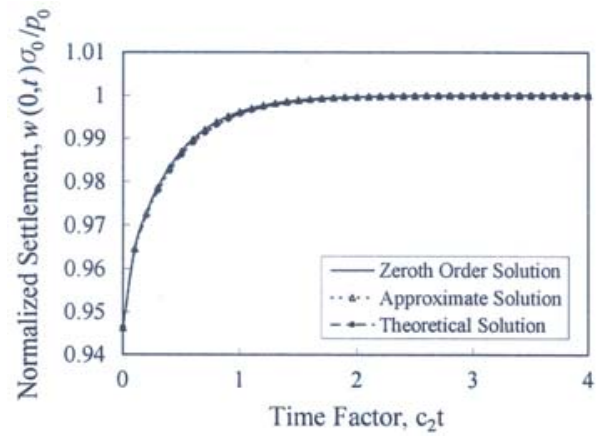

Figure 4(b). Comparison of normalized settlement of case $2: v=0.25, v_{u}=0.27, B=0.51 ; \varepsilon=\sigma_{0} / Q, c_{2}=1 / \alpha^{2} a \sigma_{0}$.

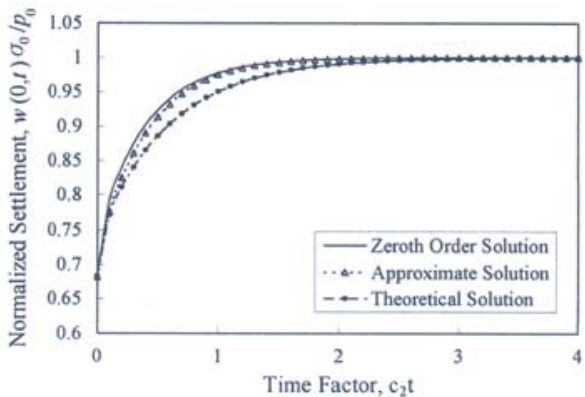

Figure 4(c). Comparison of normalized settlement of case 3: $v=0.12, v_{u}=0.31, B=0.88 ; \varepsilon=\sigma_{0} / Q, c_{2}=1 / \alpha^{2} a \sigma_{0}$.

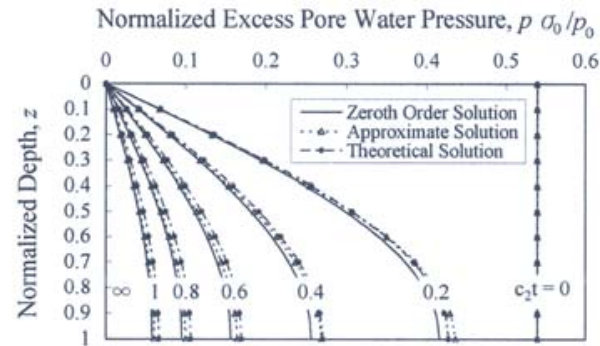

Figure 5(a). Comparison of normalized excess pore fluid pressure of case 1: $v=0.27, v_{u}=0.30, B=0.55 ; \varepsilon=\sigma_{0} / Q$, $c_{2}=1 / \alpha^{2} a \sigma_{0}$. 


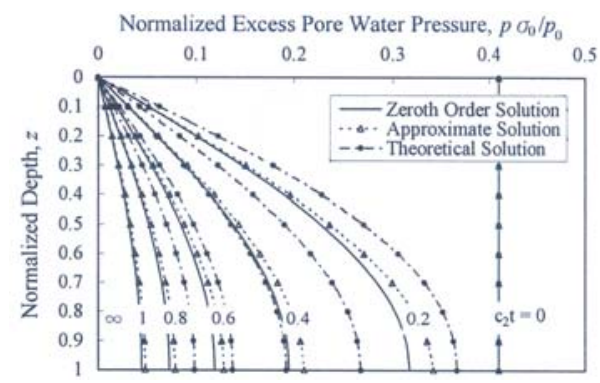

Figure 5(b). Comparison of normalized excess pore fluid pressure of case 2: $v=0.25, v_{u}=0.27, B=0.51 ; \varepsilon=\sigma_{0} / Q$, $c_{2}=1 / \alpha^{2} a \sigma_{0}$.

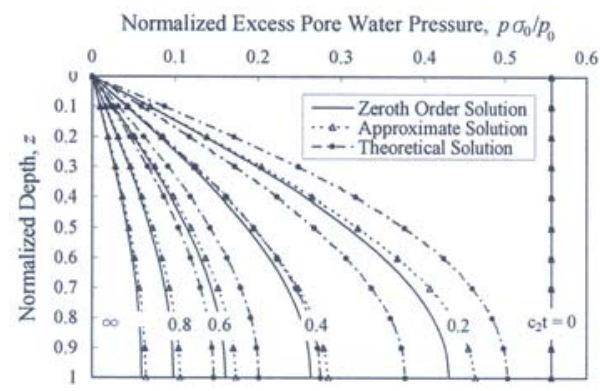

Figure 5(c). Comparison of normalized excess pore fluid pressure of case 3: $v=0.12, v_{u}=0.31, B=0.88 ; \varepsilon=\sigma_{0} / Q$, $c_{2}=1 / \alpha^{2} a \sigma_{0}$.

Based on the asymptotic expansion parameter $\varepsilon=\alpha \gamma_{w} H^{*} / G$, the comparison between the increase in time of theoretical solutions, the zeroth order solutions and approximate solutions are illustrated in Figures VI and VII, respectively. It is demonstrated that the settlement simulation results of Charcoal granite and Tennessee marble are better than Ruhr sandstone as shown in Figures VI(a), VI(b) and VI(c), respectively. Figures VII(a) to VII(c) display better simulation of excess pore pressure results of Tennessee marble than the results of Charcoal granite and Ruhr sandstone. Therefore, we can conclude that asymptotic expansion theory is a proper simulation technique on the application of consolidation deformation subjected to uniform loading.

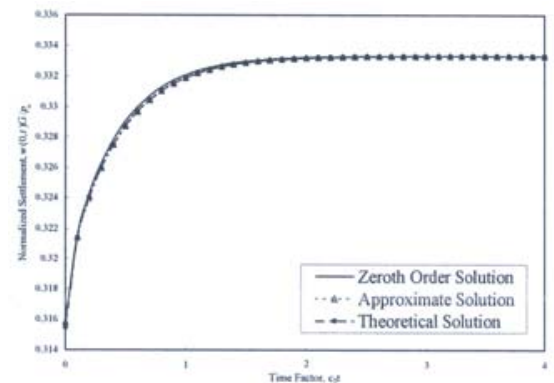

Figure 6(a). Comparison of normalized settlement of case 1: $v=0.27, v_{u}=0.30, B=0.55 ; \varepsilon=\alpha \gamma_{w} H^{*} / G, c_{3}=Q / \gamma_{w} H^{*}$.

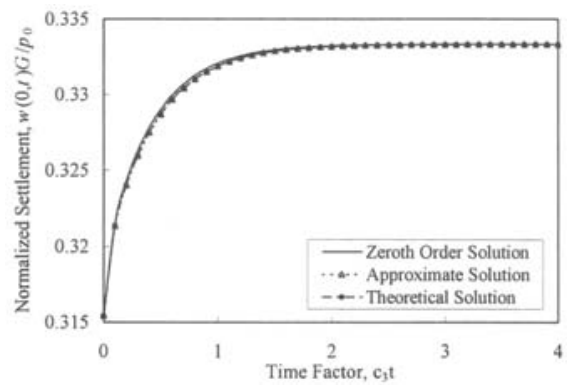

Figure 6(b). Comparison of normalized settlement of case 2: $v=0.25, v_{u}=0.27, B=0.51 ; \varepsilon=\alpha \gamma_{w} H^{*} / G, c_{3}=Q / \gamma_{w} H^{*}$. 


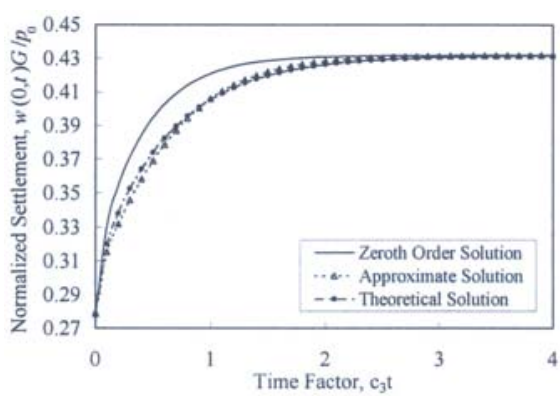

Figure 6(c). Comparison of normalized settlement of case 3: $v=0.12, v_{u}=0.31, B=0.88 ; \varepsilon=\alpha \gamma_{w} H^{*} / G, c_{3}=Q / \gamma_{w} H^{*}$

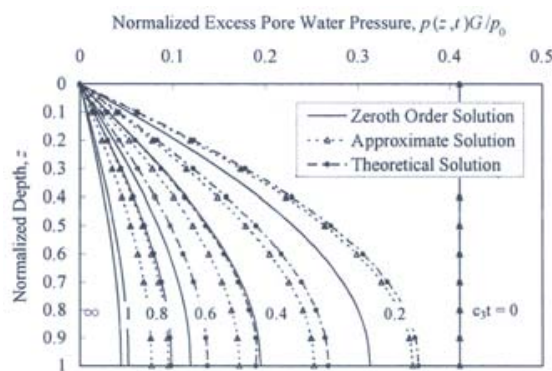

Figure 7(a). Comparison of normalized excess pore fluid pressure of case $1: v=0.27, v_{u}=0.30, B=0.55 ; \varepsilon=\alpha \gamma_{w} H^{*} / G$, $c_{3}=Q / \gamma_{w} H^{*}$.

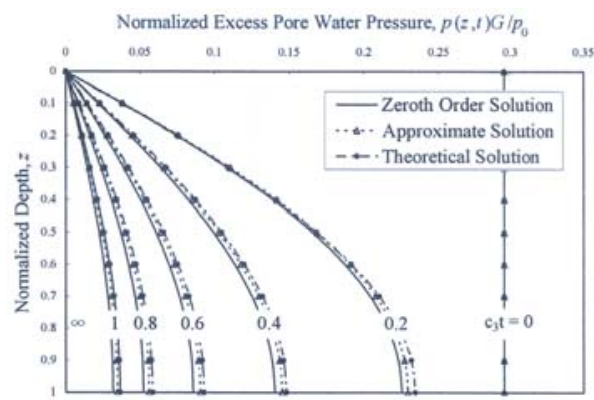

Figure 7(b). Comparison of normalized excess pore fluid pressure of case 2: $v=0.25, v_{u}=0.27, B=0.51 ; \varepsilon=\alpha \gamma_{w} H^{*} / G$, $c_{3}=Q / \gamma_{w} H^{*}$.

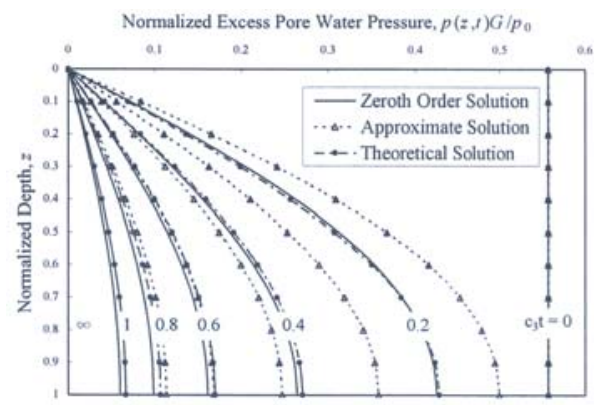

Figure 7(c). Comparison of normalized excess pore fluid pressure of case 3: $v=0.12, v_{u}=0.31, B=0.88 ; \varepsilon=\alpha \gamma_{w} H^{*} / G$,

$$
c_{3}=Q / \gamma_{w} H^{*}
$$

\section{Conclusions}

The purpose of this paper is to evaluate the asymptotic expansion theory on elastic layer of onedimensional deformation and excess pore fluid pressure subjected to uniform loading. Results from the consolidation simulations of Charcoal granite and Tennessee marble displayed better effects than the simulation results of Ruhr sandstone on the basis of three kinds of asymptotic expansion parameter. We can conclude that asymptotic expansion theory is a proper simulation method on the application 
of uniform loading induced consolidation. The results can improve understanding of the asymptotic expansion theory on coupled consolidation.

\section{Acknowledgment}

This work is supported by the Ministry of Education of Taiwan, Republic of China, through grant PEE107085.

\section{References}

[1] Z. H. Yang and J. F. Tian, "Asymptotic expansions for the gamma function in terms of hyperbolic functions,” Journal of Mathematical Analysis and Applications, vol. 478, no. 1, pp. 133-155, 2019.

[2] X. M. Huang, L. H. Cao, and X. S. Wang, “Asymptotic expansion of orthogonal polynomials via difference equations,” Journal of Approximation Theory, vol. 239, pp. 29-50, 2019.

[3] J. Barrera and H. Volkmer, “Asymptotic expansion of the $\mathrm{L}^{2}$-norm of a solution of the strongly damped wave equation,” Journal of Differential Equations, vol. 267, no. 2, pp. 902-937, 2019.

[4] Z. Q. Yang, Y. Sun, J. Z. Cui, and J. G. Ge, “A three-scale asymptotic expansion for predicting viscoelastic properties of composites with multiple configuration,” European Journal of Mechanics A/Solids, vol. 76, pp. 235-246, 2019.

[5] H. C. Kang, "Efficient calculation and asymptotic expansions of many different oscillatory infinite integrals,” Applied Mathematics and Computation, vol. 346, pp. 305-318, 2019.

[6] L. T. Hoang and V. R. Martinez, "Asymptotic expansion for solutions of the Navier-Stokes equations with non-potential body forces," Journal of Mathematical Analysis and Applications, vol. 462, no. 1, pp. 84-113, 2018.

[7] X. F. Yang, “Asymptotic expansions for Laplace transforms of Markov processes,” Journal of Mathematical Analysis and Applications, vol. 457, no. 1, pp. 694-721, 2018.

[8] Z. Q. Yang, Z. W. Hao, Y. Sun, Y. Z. Liu, and H. Dong, "Thermo-mechanical analysis of nonlinear heterogeneous materials by second-order reduced asymptotic expansion approach," International Journal of Solids and Structures, in press, 2019.

[9] J. R. Rice, and M. P. Cleary, "Some basic stress diffusion solutions for fluid-saturated elastic porous media with compressible constitutents,” Reviews of Geophysics and Space Physics, vol. 14, no. 2, pp. 227-241, 1976.

[10] M. A. Biot, “General theory of three-dimensional consolidation,” Journal of Applied Physics, vol. 12, no. 2, pp. 155-164, 1941.

[11] M. Kurashige, “A thermoelastic theory of fluid-filled porous materials,” International Journal of Solids and Structures, vol. 25, no. 9, pp. 1039-1052, 1989.

[12] E. Detournay and A. H.-D. Cheng, “Comprehensive rock engineering: practice, projects, analysis and design method,” Fundamentals of Poroelasticity, vol. 2, pp. 113-171, 1993. 